\title{
Shock effect of COVID-19 infection on environmental quality and economic development in China: causal linkages (Health Economic Evaluation)
}

\author{
Shengxia Xu ${ }^{1} \cdot$ Qiang Liu ${ }^{1} \cdot$ Xiaoli $\mathrm{Lu}^{2}$
}

Received: 1 September 2020 / Accepted: 4 September 2021 / Published online: 15 September 2021

(c) The Author(s), under exclusive licence to Springer Nature B.V. 2021

\begin{abstract}
Since coronavirus disease 2019 (COVID-19) was first reported on December 2019 in Wuhan, it fast spread to the rest of China, which has turned into a global public health problem later and generated global stock markets to violently shake. We inspect the causal relationships between economic development (ED) and environmental quality (EQ) during the period from January 2019 to May 2020 with the structural break for China and investigate the causal linkages between ED and EQ in subgroup of before and after the outbreak of COVID-19 with a semi-parametric model. The empirical tests show that smoothing structural transforms matter for the linkages of causality between ED and EQ, especially after COVID-19 infection. While the Toda-Yamamoto causality analysis supports unidirectional causality between ED and EQ before the outbreak of COVID-19, under structural shifts by the causality supplies of bidirectional casual linkages after the outbreak of COVID-19. Our results further clarified the proof that the economic activity gives rise to the environmental pollution and energy utilization mainly via the shock of COVID-19 in China. The emphasis on nonlinear causality between economic development and environmental quality may be an opportunity for China's economic recovery under considering the factor of COVID-19 infection.
\end{abstract}

Keywords COVID-19 $\cdot$ Environmental quality $\cdot$ Economic development $\cdot$ Casual linkages · Semi-parametric additive model $\cdot$ China

Qiang Liu

cuebliuqiang0910@126.com

Shengxia $\mathrm{Xu}$

cuebxushengxia@163.com

Xiaoli Lu

luxiaolicueb@163.com

1 School of Statistics, Capital University of Economics and Business, Beijing 100070, China

2 Institute of Fundamental and Interdisciplinary Sciences, Beijing Union University, Beijing 100101, China 


\section{Introduction}

The urgency and uncertainty specific to major public emergencies have brought great challenges to the development of the global economy. Since the inexplicable pneumonia was first covered on December 2019 in Wuhan, it quickly spreads to other parts of China, which has become a global public health problem later, and has result global stock markets in violent shake (Chen et al., 2020; Zhu et al., 2020). The coronavirus is originated from zoonotic diseases and associated with lethal diseases (Zaki et al., 2012); as testified by previous literatures, COVID-19 is caused by the rock-ribbed acute respiratory syndrome coronavirus 2 (SARS-CoV-2) (Sohrabi et al., 2020).

In order to get command of the diffusion of COVID-19, a mass of researches have been organized by scholars to dig out key factors influencing the transmission of SARS-CoV-2 (Zhu et al., 2020). Wang et al. (2020) have proved that human-to-human switch of COVID19 through direct contact or droplets; Kraemer et al. (2020) have examined a significant effect from population mobility to COVID-19 infection; Xie and Zhu (2020) have explored a connection between retention temperature and COVID-19's infection. On May 31, 2020, it has shown that $>83,017$ confirmed individuals have been recognized and $>4634$ deaths in the whole of China from the data of the National Health Commission; however, the number of new cases in a single day for a month has not exceeded 20 and new deaths in half a month has not increased, fortunately. In addition, other countries and regions are increasingly affected by COVID-19.

Affected by the COVID-19 infection, China's economy actually declined by $6.8 \%$ yearon-year in the first quarter of 2020, and this is the first quarterly negative growth since 1992, far exceeding the impact of 2008 international financial crisis. Recently, fighting with COVID-19 is the most pivotal challenge for whole China, which has aroused wide international concern as well (Shahzad et al., 2020). Previous literature has found that surrounding air pollutants are potential factors for respiratory infections, and they have carried out verification from different aspects (Cai et al., 2007; Xu et al., 2016). Moreover, making a thorough inquiry about the impact of air pollution with the infection of COVID-19 is interesting, especially since COVID-19 is a respiratory disease (Yang et al., 2020; Zhu et al., 2020). However, as found by existing researches, they have centralized on the relationship of air pollutants and COVID-19-confirmed numbers only, but paid no attention to the impact of causality between air pollution and COVID-19 infection. Although a nonlinear relationship between the number of accumulated confirmed cases totally and temperature was considered with COVID-19, the structural breaks were not taken into account in the nonlinear research framework.

Concerning the connection between health security and economic development, the results are quite ambiguous. Some studies support that the relationship between social security and economic development has been gradually strengthened in the process of mutual adaptation and general development in China (Li et al., 2021; Liang \& Zhang, 2019). Some studies dig out the relationship between environmental quality and economic development from the different indicators of environmental quality. Zhao and Zhou (2021) construct a four-sector endogenous economic growth model to reveal the effects of human health on economic growth under the constraint of environment pollution. Xu et al. (2021) assess $\mathrm{PM}_{2.5}$-related health and economic benefits of the Blue Sky Protection Campaign in the Beijing-Tianjin-Hebei region; they find that the reduced $\mathrm{PM}_{2.5}$ concentration will save health expenditures and avoid economic loss. Le and Nguyen (2019) advocate that energy security has a negative impact on economic growth 
when it measured by energy intensity and carbon intensity variables, under an extended version of the Cobb-Douglas production function for a global sample of 74 countries. And some studies examine the relationship between environmental quality and economic development from the different methods. Ajibike et al. (2021) take mesomeric effect model into framework of studying the relationship between environmental quality and economic development. Chege and Wang (2020) employ partial least squares regression and correlation models to dig out the linear relations between the two. In addition, Liu et al. (2021) point out that the threshold effect of air pollution on the spread of COVID-19 infection is existed, and the increase of air pollution will greatly intensify the newly confirmed cases of COVID-19 before the threshold is reached, but this aggravating effect will be weakened after the threshold. The above studies show that different types of environmental quality are negative or nonlinear related to economic development, significantly. However, they do not go beyond presenting partial causal relationships in a regression framework on the one hand. On the other hand, they ignore the importance of smoothing structural transforms for the improvement of causality framework.

The COVID-19 infection has disrupted economies and altered the lives of individuals. Many studies have shown that the incidence of the risk factors associated with the development of the disease can also be caused by a long exposure to air pollution (Guo et al., 2020; Saeha et al., 2020). Accordingly, previous studies have suggested that ambient air pollutants are risk factors for respiratory infection by carrying microorganisms to make pathogens more invasive to humans and affecting body's immunity to make people more susceptible to pathogens (Liu et al., 2021; Xu et al., 2020). Hence, it is meaningful to discuss economic development and environmental pollution, and it is also necessary to consider the impact of COVID-19 infection between them, especially since the outbreak. More importantly, considering our topic of environmental quality and economic development, no serious attempt has been made to research on a causal linkage. And reverse causality is likely to be a source of endogeneity if national economic development affects environmental quality. Therefore, the main contribution of our study is to analyze the shock effect of COVID-19 infection on environmental quality and economic development within a causal framework.

In this study, we analyze the impact of environmental quality (EQ) on economic development (ED) under the shock of COVID-19 infection using a casual linkage approach, where the approximation of Granger causality for the topic is constructed by the Fourier approximation. Concerning that economic markets and environmental sector interact with each other and discovering the economics-environment connection is of great importance to China, especially after the outbreak of COVID-19 infection with the shift process. We examine the direction of causality with Fourier approximations among ED, EQ, and energy production (EP) at first. Secondly, we take nonlinear effects into the discussion under the shock of COVID-19, via the semi-parametric additive model. Thirdly, the relationship between ED and EQ is further analyzed by measuring the direct and indirect effects of COVID-19.

The objective of this work is to assess the influence of EQ on ED under the shock of COVID-19 infection and provide a foundation for the control of environmental quality and the orderly recovery of the economy during the epidemic. The major contributions for our study are: (1) inspecting the relationship between EQ and ED under the shock of COVID19 infection for the first time; (2) carrying out the relationship between EQ, EP and ED in a causal framework of Toda-Yamamoto and extending it with a Fourier approximation innovatively; (3) expanding and revealing the relationship between ED and EQ from the 
Table 1 Basic description of the variables

\begin{tabular}{llllllrr}
\hline Variables & Unit & Indicator interpretation & Samples & Min & Max & Mean & Std.dev \\
\hline FCPI & $\%$ & Food consumer price index & 17 & 100.7 & 121.9 & 111.96 & 6.63 \\
$\mathrm{COP}$ & $\mathrm{kt}$ & Crude oil production & 17 & 1553.4 & 1656.3 & 1607.79 & 30.24 \\
$\mathrm{GNP}$ & $\mathrm{bm}^{3}$ & Natural gas production & 17 & 135.2 & 168.6 & 149.79 & 10.35 \\
$\mathrm{PG}$ & $\mathrm{bkwh}$ & Power generation & 17 & 5440.2 & 6682.4 & 5885.55 & 396.81 \\
$\mathrm{AQI}$ & $\%$ & Air quality index & 510 & 63.53 & 101.3 & 78.94 & 10.33 \\
$\mathrm{PM}_{2.5}$ & $\mu \mathrm{g} / \mathrm{m}^{3}$ & - & 510 & 22 & 79 & 43.45 & 17.46 \\
$\mathrm{PM}_{10}$ & $\mu \mathrm{g} / \mathrm{m}^{3}$ & - & 510 & 61.5 & 169.5 & 110.74 & 31.36 \\
$\mathrm{SO}_{2}$ & $\mu \mathrm{g} / \mathrm{m}^{3}$ & - & 510 & 7.67 & 17 & 12.14 & 2.76 \\
$\mathrm{CO}$ & $\mathrm{mg} / \mathrm{m}^{3}$ & - & 510 & 0.69 & 1.16 & 0.84 & 0.15 \\
$\mathrm{NO}_{2}$ & $\mu \mathrm{g} / \mathrm{m}^{3}$ & - & 510 & 25.67 & 53 & 38.98 & 8.3 \\
$\mathrm{O}_{3}$ & $\mu \mathrm{g} / \mathrm{m}^{3}$ & - & 510 & 41.67 & 139 & 93.31 & 34.03 \\
$\mathrm{NND}$ & $\mathrm{Count}$ & Number of new diagnoses & 137 & 1 & 95 & 46.96 & 29.14 \\
$\mathrm{NCD}$ & $\mathrm{Count}$ & Number of cumulative diagnoses & 137 & 291 & 83,017 & $69,167.03$ & $25,615.46$ \\
\hline
\end{tabular}

perspective of nonlinear association and providing suggestions for the orderly recovery of economic development.

\section{Methods and data}

It is worth noting that in the context of a novel casual linkage method, this is the first analysis on economic development (ED), environmental quality (EQ) and energy production (EP) with the shock of COVID-19. We inspect the causal relationships between environmental quality, economic development, and energy production in this study. What we care about is whether these causal linkages have changed, especially after an outbreak of COVID-19 infection. The assumption here is that environmental quality, economic development, and resource distribution variables will change as the structure shifts.

\subsection{Indicators' design and data description}

In light of the existing literature about environmental pollution, economic development, and energy production, considering the availability of data, 30 main provinces in mainland China are selected to make up the study area in this paper, excluding Hong Kong, Macao, Taiwan and Tibet. While for COVID-19 infection, we select all provinces in mainland China, including Hong Kong, Macao, Taiwan and Tibet. The data for all the required variables are retrieved from the National Health Commission of the People's Republic of China from January 20, 2020 to May 31, 2020, the China National Environmental Monitoring Centre of January 2019 to May 2020, the National Bureau of Statistics of January 2019 to May 2020, and the relevant government report for Chinese provinces. The descriptive statistical analysis of all variables is shown in Table 1, and we design the selected variables as follows:

Environmental quality (EQ): air pollution is treated as one of the most urgent environmental and health issues for human beings, especially for the rapid-growing developing countries such as India and China (Wakeel et al., 2016). The large number of air pollution 


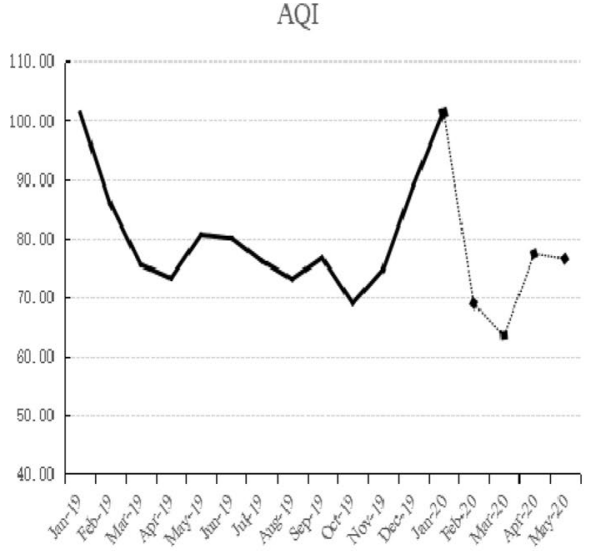

(a)

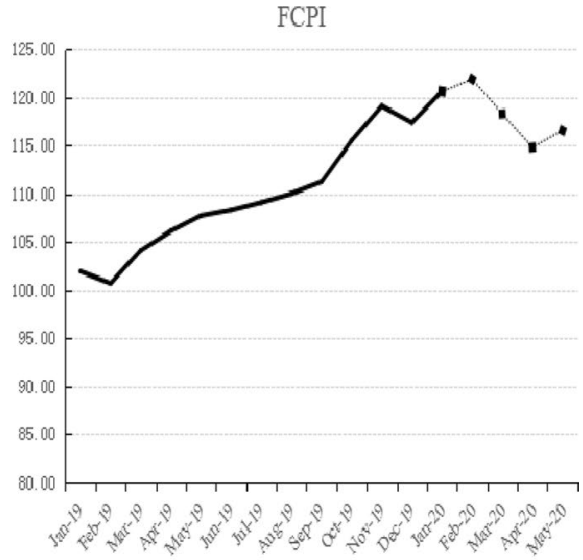

(b)

Fig. 1 The trend of AQI and FCPI. Notes: the solid line is before January 2020, which is before the outbreak of COVID-19 infection in China, and the dashed line is after the month

indicators used in existing studies was $\mathrm{CO}, \mathrm{NO}_{2}, \mathrm{SO}_{2}, \mathrm{PM}_{2.5}, \mathrm{PM}_{10}$, and $\mathrm{O}_{3}$ (Shi et al., 2017; Zhang et al., 2018), but $\mathrm{PM}_{2.5}$ was considered to be the most appropriate indicator of air pollution (Xiao et al., 2019). It should be emphasized that the above six indicators can be used to measure different aspects of air pollution only, the best way is to make up them together. More important, air quality index (AQI) can characterize the above six aspects effectively. Therefore, we choose the air quality index to characterize the EQ. It should be emphasized that the national AQI is averaged from the measured values of 30 major cities. ${ }^{1}$ In Fig. 1a, the national AQI shows a trend of fluctuations, especially after Jan 2020, compared with the same month of the previous year, the decline rate has increased significantly. And the specific statistical indicators of FPCI are shown in Table 1.

Economic development (ED): It represents the high-quality economic development including quality and quantity, not just quantitative growth, which can reflect the economic development scale of a country or region, and it is characterized by GDP per capita as usual (Balcilar et al., 2010; Durusu-Ciftci et al., 2020). However, the publication of GDP in China is mainly based on quarterly and annual data; the daily or monthly data required in this article is not easy to obtain. Nevertheless, consumer price index (CPI) is closely related to the lives of the people and has an important position in the entire national economic price system. Considering that the indicator can reflect the economic development and can be obtained, food consumer price index (FCPI) is used to portray ED. In Fig. 1b, FCPI shows a rising trend monthly, although it has rebounded after February 2020, comparing with the same period of the previous year, it has increased significantly. And the specific statistical indicators of FPCI are shown in Table 1.

Energy production (EP): It is obvious that the advancement of China's economy is touching the peak in the world in fact. Meanwhile, due to industrial production, construction, urbanization, transportation, trade, service industry and many other sectors depending

\footnotetext{
130 major cities are Beijing, Shanghai, Tianjin, Chongqing, Hangzhou, Harbin, Changchun, Shenyang, Shijiazhuang, Taiyuan, Xian, Jinan, Urumqi, Lhasa, Xining, Lanzhou, Yinchuan, Zhengzhou, Nanjing, Wuhan, Hefei, Fuzhou, Nanchang, Changsha, Guiyang, Chengdu, Guangzhou, Kunming, Nanning, Shenzhen.
} 

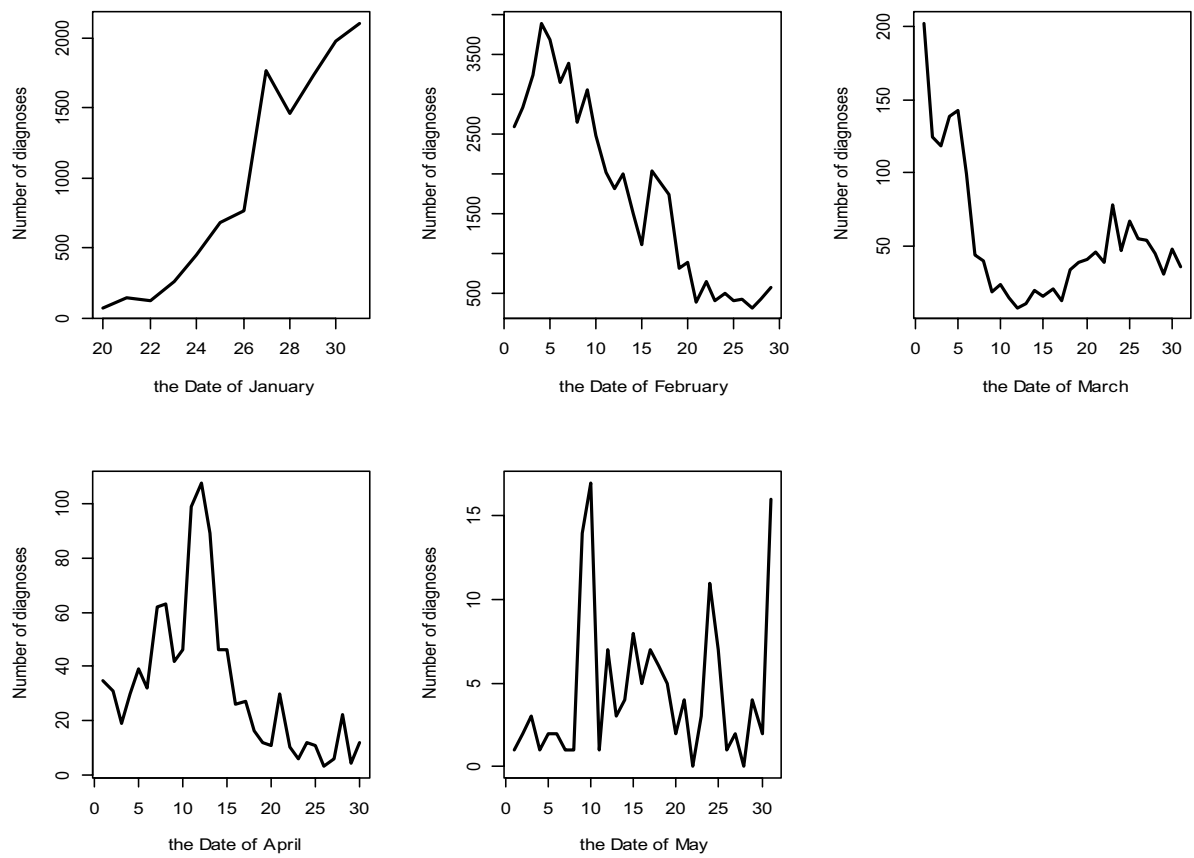

Fig. 2 Numbers of new diagnoses of COVID-19 infection in China

on energy consumption profoundly, China is the one of largest energy consumers around the world (Liu et al., 2018; Rauf et al., 2018). From an input-output perspective, energy consumption is as important as production and is a key indicator of the current energy situation. Therefore, crude oil production (COP), natural gas production (GNP), and power generation (PG) are used to calculate the comprehensive energy production via the equal weighting method.

COVID-19 infection (CI_19): Daily confirmed new cases are considered to be the most direct effective indicator of COVID-19 infection in most researches (Ma et al., 2020; Zhu et al., 2020). The number of new diagnoses (NND) for every city from January 20, 2020, to May 31, 2020, was received from the reports released by local health commissions on the official websites. We make partial corrections to national data based on data from various provinces. In Fig. 2, since the data were collected on January 20, 2020, China's COVID-19 infection has experienced a large increase, followed by a continuous decrease, and then a relatively stable trend of small changes; of which, on February 4, 2020, the number of new diagnoses reached the maximum (3887). In addition, in the application of the causal linkage model in case analysis, we refer to the monthly data of COVID-19 infection and select the number of cumulative diagnoses (NCD) on the first and last day of each month as a subtraction.

\subsection{Granger causality with smooth structural shifts}

Granger causality is a test method that tries to reveal the influence relationship between variables. And the standard Granger causality analysis necessitates testing for unit root and 
co-integration because Wald test not only has a non-standard distribution if the variables in VAR model are integrated, but also depend on nuisance parameters. However, Toda and Yamamoto (1995) have improved that Granger causality overcomes these problems by estimating VAR model; in addition, they used it to study the nonlinear and non-unidirectional relationship between variables.

There are many ways to explain structural cuts. An ordinary approach is put forward by Nazlioglu et al. (2016) including abrupt and gradual in causality study and carried forward by Durusu-Ciftci et al. (2020). And then, it is augmented by a Fourier approximation within the Toda-Yamamoto framework. In their studies, the constant terms are variable over time and defined as $\alpha(t)$ after the constant assumption has relaxed by an unknown time with the gradual structural shifts. Therefore, the numbers and forms of cuts are captured by the Fourier approximation, which can be defined as:

$$
\alpha(t) \cong c_{0}+\sum_{k=1}^{K} \alpha_{1 k} \sin \left(\frac{2 \pi t k}{T}\right)+\sum_{k=1}^{K} \alpha_{2 k} \cos \left(\frac{2 \pi t k}{T}\right)
$$

where $\alpha(t)$ are time-dependent and present structural shifts in $y_{\mathrm{t}}, K$ is the frequency's number, $\alpha_{1 \mathrm{k}}$ can calculate the amplitude of the frequency, and $\alpha_{2 \mathrm{k}}$ can compute the displacement of the frequency. A Fourier approximation takes into the Toda-Yamamoto framework that can be set:

$$
y_{\mathrm{t}}=c_{0}+\sum_{k=1}^{K} \alpha_{1 k} \sin \left(\frac{2 \pi t k}{T}\right)+\sum_{k=1}^{K} \alpha_{2 k} \cos \left(\frac{2 \pi t k}{T}\right)+\beta_{1} y_{t-1}+\beta_{2} y_{t-2}+\cdots+\beta_{p+d} y_{t-(p+d)}+\varepsilon_{\mathrm{t}}
$$

where $y_{\mathrm{t}}$ consists of endogenous variables, $c_{0}$ is a vector of constant terms, $\beta_{1}, \beta_{2}, \cdots, \beta_{p+d}$ are the matrices of coefficients, $p$ is the order of lag, $d$ is the maximum integration order of variables and $\varepsilon_{\mathrm{t}}$ are the white-noise residuals.

In this paper, we employ environmental quality (EQ), economic development (ED) and energy production (EP) as endogenous variables from the panel data, and Eq. (2) can definitely be re-defined as:

$$
\begin{aligned}
\mathrm{ED}_{\mathrm{t}}= & c_{10}+\sum_{k=1}^{K} \alpha_{1 k} \sin \left(\frac{2 \pi t k}{T}\right)+\sum_{k=1}^{K} \alpha_{2 k} \cos \left(\frac{2 \pi t k}{T}\right) \\
& +\sum_{j=1}^{p+d} \alpha_{1 j}^{*} \mathrm{ED}_{t-j}+\sum_{j=1}^{p+d} \beta_{1 j}^{*} \mathrm{EQ}_{t-j}+\sum_{j=1}^{p+d} \gamma_{1 j}^{*} \mathrm{EP}_{t-j}+\varepsilon_{1 \mathrm{t}} \\
\mathrm{EQ}_{\mathrm{t}}= & c_{20}+\sum_{k=1}^{K} \alpha_{1 k} \sin \left(\frac{2 \pi t k}{T}\right)+\sum_{k=1}^{K} \alpha_{2 k} \cos \left(\frac{2 \pi t k}{T}\right) \\
& +\sum_{j=1}^{p+d} \alpha_{2 j}^{*} \mathrm{ED}_{t-j}+\sum_{j=1}^{p+d} \beta_{2 j}^{*} \mathrm{EQ}_{t-j}+\sum_{j=1}^{p+d} \gamma_{2 j}^{*} \mathrm{EP}_{t-j}+\varepsilon_{2 \mathrm{t}}
\end{aligned}
$$




$$
\begin{aligned}
\mathrm{EP}_{\mathrm{t}}= & c_{30}+\sum_{k=1}^{K} \alpha_{1 k} \sin \left(\frac{2 \pi t k}{T}\right)+\sum_{k=1}^{K} \alpha_{2 k} \cos \left(\frac{2 \pi t k}{T}\right) \\
& +\sum_{j=1}^{p+d} \alpha_{3 j}^{*} \mathrm{ED}_{t-j}+\sum_{j=1}^{p+d} \beta_{3 j}^{*} \mathrm{EQ}_{t-j}+\sum_{j=1}^{p+d} \gamma_{3 j}^{*} \mathrm{EP}_{t-j}+\varepsilon_{3 \mathrm{t}}
\end{aligned}
$$

where $t(t=1,2, \ldots, T)$ is the $t$-th period, the null hypothesis $H_{0}$ of Granger with noncausality is on account of zero restrictions on the first $p$ parameters in the framework of Toda-Yamamoto causality, and it has an asymptotic Chi-square distribution with $p$ degrees of freedom $\chi^{2}(p)$ by conducting the Wald statistic. However, because the value of frequency $k$ is variable, an asymptotic Chi-square distribution of the Wald statistic may not be satisfied to a great extent. In order to avoid the above problem, an approach of sampling bootstrap from residual has been put forward by Efron (1979) with the bootstrap distribution of Wald statistic. This method has been widely used by decreasing the $p$ value of the tests with small samples, as well as being robust to the unit root and co-integration properties of the empirical analysis in recent works in particular (Balcilar et al., 2010; DurusuCiftci et al., 2020). Therefore, the Wald statistics are obtained from bootstrap distribution in this paper.

The most important problem in Eqs. (3), (4), and (5) is that it needs to confirm the value of cumulative Fourier frequency $K$ and lag lengths $p$. In order to ascertain the optimal number of lagging orders in a causality framework, it is common to apply Akaike information criterion (AIC). As shown in the latest researches about Granger causality, the number of Fourier frequency and lag lengths can also be determined by AIC; therefore, following the above convention, we use AIC to determine Fourier frequency $K$ and lag lengths $p$ in this paper also.

\subsection{Nonlinear model specification}

Considering the assumption that EQ, ED, and RD have relatively complicated causal relationships, it is difficult for a simple linear model to present the complex changing between them, especially the outbreak of COVID-19 infection, making the relationships more complicated. Therefore, we use a semi-parametric model to quantitatively analyze the nonlinear interaction, due to the multiple variables involved; the semi-parametric additive model is designed:

$$
y_{\mathrm{t}}=f\left(\sum_{i=1}^{p} x_{1, t-i}\right)+f\left(\sum_{i=1}^{p} x_{2, t-i}\right)+\sum_{i=1}^{p} C I_{-} 19_{t-i}+\varepsilon_{\mathrm{t}}
$$

where $y_{\mathrm{t}}$ consists of endogenous variables, $p$ is the lag order, $f(\cdot)$ is the smooth function of independent variables to reflect its nonlinear effect on $y_{t}$, CI_19 is the indicator of COVID19 infection, and $\varepsilon_{\mathrm{t}}$ are the white-noise residuals. In terms of estimation, on the basis of Engle and Russell (1998), a semi-parametric additive model estimation method is proposed by Buhlmann and Meneil (2002), and the smooth spline functions are used for nonparametric fitting. 

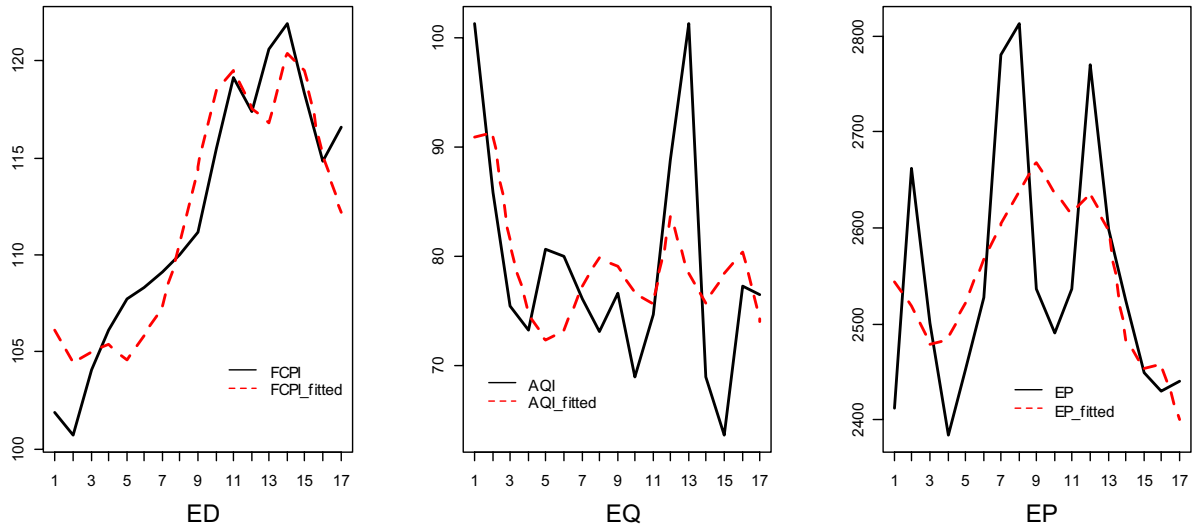

Fig. 3 The trend with Fourier approximations. Notes: the solid line is the spline fitting curve, and the dotted line is the upper and lower confidence curves of the fitting interval with $95 \%$ confidence

Table 2 Results of the unit root tests

\begin{tabular}{llll}
\hline Variables & Dickey-Fullers & Lag orders & Maximum integration order \\
\hline ED (FCPI) & 1.286 & 1 & 1 \\
EQ (AQI) & -0.469 & 1 & \\
EP (IEP) & -0.481 & 1 & \\
CI_19 (NND) & -2.324 & 1 & \\
\hline
\end{tabular}

\section{Empirical results}

Figure 3 demonstrates the food consumer price index (\%), the air quality index (\%), and the integrated energy production with their Fourier approximations. Obviously, the variations of these variables are for specific country or region as usual, and the trend will dynamic change over time. What's more, China has undergone structural changes both in the markets of environment and expenditure of energy. However, the distribution form of these changes is unknown usually and there is no priori information of choosing certain-cut dates and the number of cuts also. As shown in the figure, the Fourier approximation method can capture the long fluctuations in the series, so it seems to be more precise in controlling the unknown nature of the cutoffs in the causality framework.

In terms of Toda-Yamamoto Granger causality analysis, the first step is to inspect the unit root properties of the variables to fix the maximum order of integration $(d)$ in the VAR system. Therefore, a group of unit root tests have employed by us according to ADF test of Dickey and Fuller (1979), ADF test with one-cut situation of Zivot and Andrews (1992) and ADF test with the Fourier approximation of Enders and Lee (2012). In Table 2, the consequences show that although the null hypothesis of unit root cannot be rejected for the level variables, all unit root tests strongly support the evidence of stationarity for the first-difference variables. And then, the maximum integration order of the variables $(d)$ is verified as 1 in the $\operatorname{VAR}(p+d)$ models. 
Table 3 Direction of causal linkages from multivariate models

\begin{tabular}{|c|c|c|c|c|}
\hline \multirow[b]{2}{*}{ Relationships } & \multicolumn{2}{|c|}{$\begin{array}{l}\text { Before COVID-19 infec- } \\
\text { tion }\end{array}$} & \multicolumn{2}{|c|}{$\begin{array}{l}\text { After COVID-19 } \\
\text { infection }\end{array}$} \\
\hline & $\mathrm{TY}$ & FTY & $\mathrm{TY}$ & FTY \\
\hline ED and EQ & $\mathrm{N}$ & $\leftarrow(+)$ & $\rightarrow(-)$ & $\leftrightarrow(-,+)$ \\
\hline ED and EP & $\rightarrow(-)$ & $\leftarrow(+)$ & $\leftrightarrow(-,+)$ & $\leftrightarrow(-,+)$ \\
\hline EQ and EP & $\leftrightarrow(-,+)$ & $\leftrightarrow(-,+)$ & $\rightarrow(-)$ & $\rightarrow(-)$ \\
\hline
\end{tabular}

TY: the TY approach which does not consider the structural breaks. FTY: the Fourier TY approach with cumulative frequencies is based on Eq. (2). N: there has no causality between the two. $\leftrightarrow:$ there is a mutual influence between the two. $\rightarrow:$ the former does cause the later. $\leftarrow$ : the later does cause the former. $(-)$ : there is a negative relation between the two. $(+)$ : there is a positive relation between the two

The second step of the Toda-Yamamoto Granger causal framework is to test the existence of causal linkages in multivariate model that all three variables included in the VAR estimations. The outcomes from the Toda-Yamamoto (TY) and Fourier Toda-Yamamoto (FTY) causality analyses are displayed in Tables 3, and while COVID-19 infection as the boundary, the test of causality is divided into two parts. Moreover, before proceeding with the inference of causal analysis, we use F-test to examine the significance of the Fourier terms in all estimated VAR models. In most cases, the null hypothesis of the absence of Fourier terms is rejected, which implies the importance of smoothing structural changes.

In Table 3, the outcomes of causality between ED and EQ, Toda-Yamamoto test suggest no causality in any direction between ED and EQ during before COVID-19 infection time, while there is a unidirectional causal relationships from EQ to ED when structural breaks are taken into account. However, the results for causal linkages between ED and EQ are stronger after COVID-19 infection whether it is in Toda-Yamamoto test framework or in Toda-Yamamoto test framework with Fourier approximations; moreover, causal linkage presents bidirectional causal relationships between ED and EQ when structural breaks are taken into account. After the outbreak of COVID-19 infection, it appears as a restraining effect between the improvement of environmental quality and the slowdown of economic development, and a positive effect between the improvement of economic development level and the deterioration of environmental quality. That means that the causal linkages between ED and EQ will not only be affected by whether structural transformation is considered, but also by COVID-19 infection.

The results for causality are between ED and EP, while there is a unidirectional causal relationships from ED to EP, a unidirectional causal relationships from EP to ED in the framework of Toda-Yamamoto test during the period of before COVID-19 infection. However, after the outbreak of COVID-19 infection, whether or not structural breaks are taken into account, there is a significant two-way causal relationships between ED and EP, and the sign of action is the same. In other words, the continuous improvement of energy production level can effectively promote the improvement of economic development level, but the further improvement of economic level can restrain the increase of energy production to some extent and then show a certain threshold effect.

Different with the results of the previous two groups of causal linkages, the results for causality between EQ and EP indicate bidirectional causal relationships no matter the structural shifts are taken into account or not during the period of before the outbreak of 
COVID-19 infection. On the contrary, after COVID-19 infection, there are unidirectional causal linkages from EQ to EP in both Toda-Yamamoto test and Toda-Yamamoto test with Fourier approximations, and it shows a negative effect. We find that the deepening of environmental pollution will greatly reduce energy production capacity; in other words, the improvement of energy production capacity is inseparable from the effective improvement of environmental quality.

In general, we can conclude as: (i) considering structural shifts have an important impact on economic-energy, economic-environment and environment-energy connections, (ii) the causal relationships are mostly sensitive to structural shifts in the economic-environment relation, (iii) the shock effect of COVID-19 infection has an important role on the causal acting among environmental quality, economic development and energy production. Hence, (i) policy implications may differ according to the causality frameworks with or without structural shifts and (ii) policy implications may differ according to the outbreak of COVID-19 infection.

\section{Additional issues}

In this paper, there are two more issues to better dig out the causal relationships among environment, economics and energy under the shock of COVID-19 infection. First, the univariate linear model setting is the simplest and most direct way to test whether there is an influence linkage between the independent variable and the dependent variable. For the purpose of finding the linear impact of COVID-19 infection on environment-economic-energy systems, we take COVID-19 infection (CI_19) as the independent variable, and economic development (ED), environmental quality (EQ) and energy production (EP) as the explanatory variable, respectively.

The results for the linear shock impact of COVID-19 infection on ED, EQ and EP are shown in Table 4. In terms of ED, we find that there is a positive relationship from CI_19 to food consumer price index (FCPI), and the increase of the number of new diagnoses (NND) will increase the value of FCPI, which can reflect the state of economic development, manifested by rising prices and sluggish economic development. In terms of EQ, although the parameter does not pass the significance test, we examine the significantly negative association from CI_19 to air quality index (AQI). As demonstrated by existing literature, air pollution is a key factor in COVID-19 infection, for example, $\mathrm{SO}_{2}$ was correlated with the number of confirmed cases per day negatively (Zhu et al., 2020); a significant correlation of atmospheric particulate matter $\left(\mathrm{PM}_{2.5}\right.$ and $\left.\mathrm{PM}_{10}\right)$ and hospitalizations for respiratory disease by using a distributed lag nonlinear model (Xie et al., 2019). In summary, all of the six air pollutants could be risk factors in respiratory infection. However, our findings require further study. In terms of EP, we discover that there is a negative relationship from CI_19 to comprehensive energy production (CEP), but the coefficient does not pass the significance test. In summary, the linear impact of COVID-19 infection on ED, EQ and EP is not very significant, but it can provide a basis for promoting the improvement of China's economic development level.

Another issue is to consider the nonlinear effect to further deep the causal linkages. In Eq. (6), the lag order can be determined as 1 from the results in Table 2; combining the outcomes of causality (in Table 3), we make further estimation and analysis of the semiparametric additive models between economic development and environmental quality, 


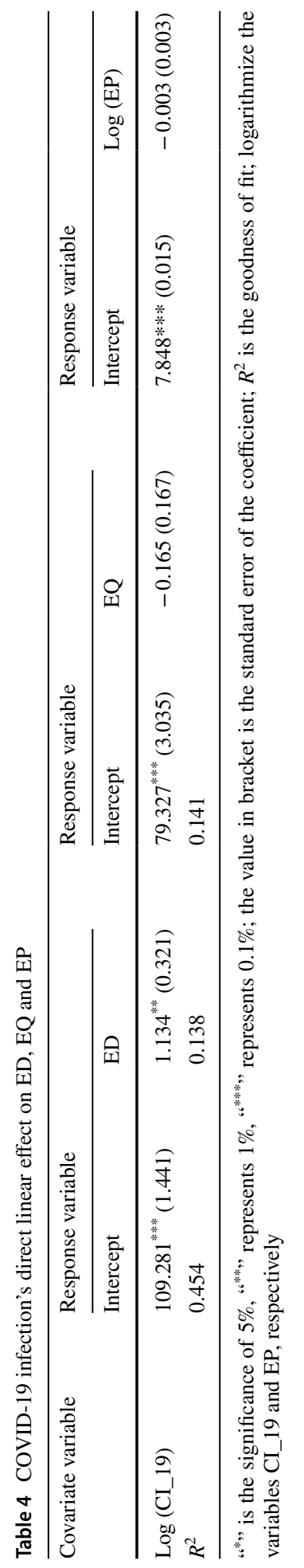




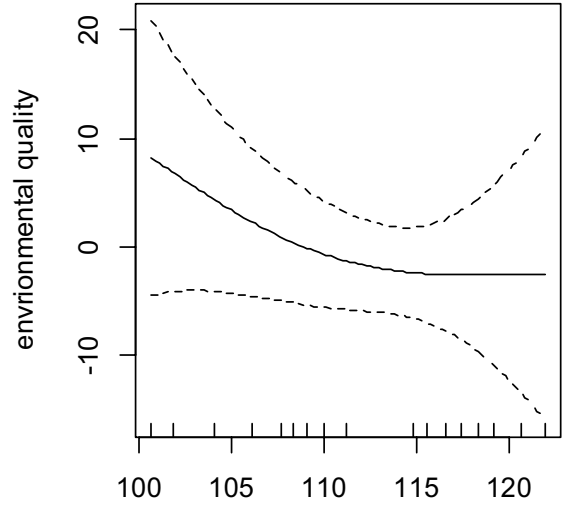

economic develoment

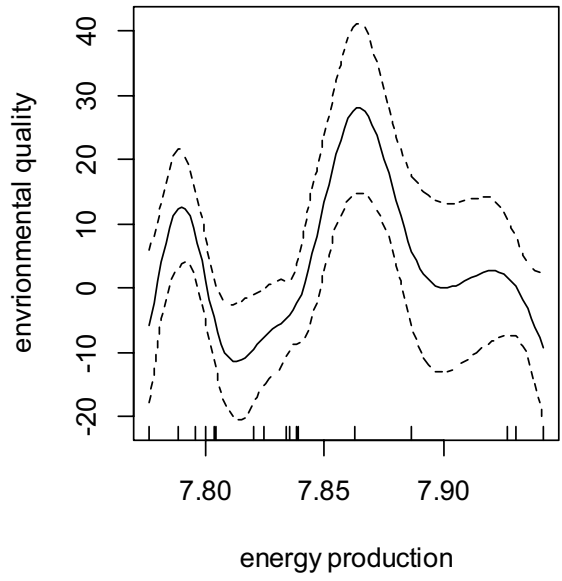

Fig. 4 The nonlinear casual linkages under the shock of COVID-19 infection. Notes: the black solid line are the connection of the original values of the three variables, and the red dotted lines are the connection of the fitted values of the three variables with Fourier approximations

between energy production and environmental quality, with the number of new diagnoses (NND) as the parameter part.

Figure 4 presents the results for the nonlinear shock effect under COVID-19 infection, the $p$ values of approximate significance of smooth terms are 0.042 and 0.034 , respectively. Meanwhile, on the one hand, considering the COVID-19 infection of the food consumer price index has impact on the air quality index positively, which means that the reduction in the number of new diagnoses will significantly improve the environmental quality; on the other hand, COVID-19 infection, which considers the comprehensive production of energy, has a negative effect on the air quality index. In addition, we examine the nonlinear relationships between ED and EQ and between EP and EQ; the outbreak of COVID-19 infection has a certain impact on China's economic development, environmental quality, and energy production capacity, obviously.

Our study has some implications for the recovery of China's economy and the improvement of people's living standards under the impact of COVID-19. First, the government and the public should pay more attention to areas with high AQI and low EP, because these areas may suffer from a more severe COVID-19 epidemic, and these areas may need to recover their economy from the aspects of price index adjustment and energy consumption. Second, it is worth noting that the nonlinear causal relationships among environmental quality, economic development and energy production are limited by the small amount of data and only consider the bivariate causal framework, and further laboratory research is needed to clarify their underlying mechanism.

\section{Conclusions}

This study testifies the causal associations between economic development and environmental quality during the period January 2019 to May 2020 from China. China has experienced either structural changes rapidly or reforms smoothly in economic, energy and environment systems during the period, especially after COVID-19 infection. For 
the purpose of testing the character of structural changes in causal relationships, the conventional Toda-Yamamoto inspection is employed at first and then the Fourier Toda-Yamamoto approximations. Moreover, we explore the linear and nonlinear impact effects of COVID-19 infection on economic development and environmental quality.

Our results show that structural changes are important for discovering causality, and linkages between economic development and environmental quality are mostly sensitive to structural changes firstly. Secondly, the proof that the economic activity gives rise to the environmental pollution and energy utilization mainly via the shock of COVID19 is further clarified in China. In other words, the outbreak of COVID-19 infection is the main reason for the slowdown in China's economic development and the decline of environmental quality since January 2020. Thirdly, the outbreak of COVID-19 infection has a certain impact on society's development, and the reduction in the number of new diagnoses will significantly improve the environmental quality, increase energy production capacity, and promote economic recovery and development. Moreover, the emphasis on nonlinear causality between economic development and environmental quality may be supplied an opportunity for China's economic recovery under considering the factor of COVID-19 infection.

Based on the above research findings, the following policy recommendations are given: policymakers need to create a conducive environmental quality through virus transmission controlling and increase vaccination efforts at first. It has proved that the control of the spread of the virus depends on excellent environmental quality, and attention to environmental quality can provide support for suppressing the spread of the virus; meanwhile, vaccination can improve immunity, which has reached a consensus in countries around the world. Second, the government and the public should pay more attention to areas with high environmental quality and low energy production, because these areas may suffer from a more severe COVID-19 epidemic. Judging from the evolution of the current epidemic, these regions generally have a relatively large flow of population, especially during holidays. Once an epidemic is discovered, its spread speed and scale are relatively large, so attention needs to be paid and the flow of population needs to be controlled. Thirdly, economic development can be promoted by improving environmental quality and reducing energy consumption, but the nonlinear causal relationships between environmental quality and economic development cannot be ignored. From the perspective of China's economic development, rapid economic development is often accompanied by environmental pollution and energy consumption; meanwhile, improving environmental quality and reducing energy consumption can promote the quality of economic development. Therefore, the causal relationship between them should be further explored.

Our study has several limitations. First, our data did not include daily records of economic and environmental indicators, so we could not conduct a comprehensive and detailed analysis. Second, we focused on the causal effect of economic development and environmental quality under the shock of COVID-19 infection and did not control the impact of other potential variables. Third, we gave the possible research ideas on the nonlinear causality in economy-environment-energy systems under the shock of COVID-19 infection, but due to data limitations, we did not conduct verification studies. Fourth, because the cities in other countries or regions are not included in this study, our findings are not globally representative. Therefore, maybe we need to conduct research to overcome these limitations in future. 


\section{References}

Ajibike, W. A., Adeleke, A. Q., Mohamad, F., Bamgbade, J. A., Nawi, M. N. M., \& Moshood, T. D. (2021). An evaluation of environmental sustainability performance via attitudes, social responsibility, and culture: A mediated analysis. Environmental Challenges 4: 100161. ISSN 2667-0100. https://doi.org/10.1016/j.envc.2021.100161

Balcilar, M., Ozdemir, Z. A., \& Arslanturk, Y. (2010). Economic growth and energy consumption causal nexus viewed through a bootstrap rolling window. Energy Economics, 32, 1398-1410.

Buhlmann, P., \& Meneil, A. J. (2002). An algorithm for nonparametric GARCH modelling. Computational Statistics \& data Analysis, 4(10), 665-683.

Cai, Q.-C., Lu, J., Xu, Q.-F., Guo, Q., Xu, D.-Z., Sun, Q.-W., Yang, H., Zhao, G.-M., \& Jiang, Q.-W. (2007). Influence of meteorological factors and air pollution on the outbreak of severe acute respiratory syndrome. Public Health, 121, 258-265. https://doi.org/10.1016/j.puhe.2006.09.02

Chege, S. M., \& Wang, D. P. (2020). The impact of entrepreneurs' environmental analysis strategy on organizational performance. Journal of Rural Studies, 77, 113-125.

Chen, H., Guo, J., Wang, C., Luo, F., Yu, X., Zhang, W., Li, J., Zhao, D., Xu, D., \& Gong, Q. (2020). Clinical characteristics and intrauterine vertical transmission potential of COVID-19 infection in nine pregnant women: A retrospective review of medical records. The Lancet, 395, 809-815.

Dickey, D. A., \& Fuller, W. A. (1979). Distribution of the estimators for autoregressive time series with a unit root. Journal of the American Statistical Society, 75, 427-431.

Durusu-Ciftci, D., Soytas, U., \& Nazlioglu, S. (2020). Financial development and energy consumption in emerging markets: Smooth structural shifts and causal linkages. Energy Economics, 87, 104729. https://doi.org/10.1016/j.eneco.2020.104729

Efron, G. (1979). Bootstrap methods: Another look at the jackknife. Annals of Statistics, 7, 1-26.

Enders, W., \& Lee, J. (2012). The flexible Fourier form and Dickey-Fuller type unit root tests. Economics Letters, 117, 196-199.

Engle, R. F., \& Russell, J. R. (1998). Autoregressive conditional duration: A new model for irregularlyspaced transaction data. Econometrica, 66, 1127-1162.

Guo, Y.-R., Cao, Q.-D., Hong, Z.-S., Tan, Y.-Y., Chen, S.-D., Jin, H.-J., Tan, K.-S., Wang, D.-Y., \& Yan, Y. (2020). The origin, transmission and clinical therapies on coronavirus disease 2019 (COVID-19) outbreak-an update on the status. Military Medical Research, 7, 11-17.

Kraemer, M. U., Yang, C.-H., Gutierrez, B., Wu, C.-H., Klein, B., Pigott, D. M., du Plessis, L., Faria, N. R., Li, R., \& Hanage, W. P. (2020). The effect of human mobility and control measures on the COVID-19 epidemic in China. Science. https://doi.org/10.1126/science.abb4218

Le, T. H., \& Nguyen, C. P. (2019). Is energy security a driver for economic growth? Evidence from a global sample. Energy Policy, 129, 436-451.

Li, Q., Zhao, Y., Li, S. L., \& Zhang, L. L. (2021). Spatial-temporal characteristics of the coupling coordination of social security and economic development in China during 2002-2018. Regional Sustainability, 2, 116-129.

Liang, C. C., \& Zhang, S. J. (2019). Social security expenditure efficiency corruption and economic growth: An empirical study based on China provincial data. Hubei Social Sciences, 1, 78-86.

Liu, L., Zhou, C., Huang, J., \& Hao, Y. (2018). The impact of financial development on energy consumption demand: Evidence from China. Emerging Markets Finance and Trade 54(2): 269-287.

Liu, Q., Xu, S. X., \& Lu, X. L. (2021). Association between environmental pollution and COVID-19 infection: Evidence from data at national and municipal levels. Environmental Science and Pollution Research, 28, 37231-37243.

Ma, Y., Zhao, Y., Liu, J., He, X., Wang, B., Fu, S., Yan, J., Niu, J., Zhou, J., \& Luo, B. (2020). Effects of temperature variation and humidity on the death of COVID-19 in Wuhan, China. Science of Total Environment. https://doi.org/10.1016/j.scitotenv.2020.138226

Nazlioglu, S., Gormus, N. A., \& Soytas, U. (2016). Oil prices and real estate investment trusts (REITs): Gradual-shift causality and volatility transmission analysis. Energy Economics, 60, 168-175.

Rauf, A., Zhang, J., Li, J., \& Amin, W. (2018). Structural changes, energy consumption and Carbon emissions in China: Empirical evidence from ARDL bound testing model. Structural Change and Economic Dynamics. https://doi.org/10.1016/j.strueco.2018.08.010

Shin, S., Bai, L., Oiamo, T. H., Burnett, R. T., Weichenthal, S., Jerrett, M., \& Chen, H. (2020). Association between road traffic noise and incidence of diabetes mellitus and hypertension in Toronto, Canada: A population-based cohort study. Journal of American Heart Association 9: e013021.

Shahzad, F., Shahzad, U., Fareed, Z., Najaf, I., Shujahat, H. H., \& Fayyaz, A. (2020). Asymmetric nexus between temperature and COVID-19 in the top ten affected provinces of China: A current 
application of quantile-on-quantile approach. Science of the Total Environment. https://doi.org/10. 1016/j.scitotenv.2020.139115

Shi, H., Fan, J., \& Zhao, D. (2017). Predicting household PM2.5-reduction behavior in Chinese urban areas: An integrative model of theory of planned behavior and norm activation theory. Journal of Cleaner Production, 145, 64-73.

Sohrabi, C., Alsafi, Z., O’Neill, N., Khan, M., Kerwan, A., Al-Jabir, A., Iosifidis, C., \& Agha, R. (2020). World Health Organization declares global emergency: A review of the 2019 novel coronavirus (COVID-19). International Journal of Surgery. https://doi.org/10.1016/j.ijsu.2020.02.034

Toda, H. Y., \& Yamamoto, T. (1995). Statistical inference in vector auto-regressions with possibly integrated processes. Journal of Econometrics, 66(1), 225-250.

Wakeel, M., Yang, S., Chen, B., Hayat, T., Alsaedi, A., \& Ahmad, B. (2016). Network perspective of embodied PM2.5- a case study. Journal of Cleaner Production, 142, 3322-3331.

Wang, D., Hu, B., Hu, C., Zhu, F., Liu, X., Zhang, J., Wang, B., Xiang, H., Cheng, Z., \& Xiong, Y. (2020). Clinical characteristics of 138 hospitalized patients with 2019 novel coronavirus-infected pneumonia in Wuhan, China. The Journal of the American Medical Association. https://doi.org/10.1001/jama. 2020.1585

Xiao, O. Y., Shao, Q. L., Zhu, X., He, Q. Y., Xiang, C., \& Wei, G. E. (2019). Environmental regulation, economic growth and air pollution: Panel threshold analysis for OECD countries. Science of the Total Environment, 657, 234-241.

Xie, J., \& Zhu, Y. (2020). Association between ambient temperature and COVID-19 infection in 122 cities from China. Science of the Total Environment. https://doi.org/10.1016/j.scitotenv.2020.138201

Xie, J., Teng, J., Fan, Y., Xie, R., \& Shen, A. (2019). The short-term effects of air pollutants on hospitalizations for respiratory disease in Hefei, China. International Journal of Biometeorology, 63, 315-326.

Xu, M., Qin, Z. L., Zhang, S. H., \& Xie, Y. (2021). Health and economic benefits of clean air policies in China: A case study for Beijing-Tianjin-Hebei region. Environmental Pollution. https://doi.org/10. 1016/j.envpol.2021.117525

Xu, Q., Li, X., Wang, S., Wang, C., Huang, F., Gao, Q., Wu, L., Tao, L., Guo, J., \& Wang, W. (2016). Fine particulate air pollution and hospital emergency room visits for respiratory disease in urban areas in Beijing, China, in 2013. PLoS ONE. https://doi.org/10.1371/journal.pone.0153099

Xu, Z., Shi, L., Wang, Y., Zhang, J., Huang, L., Zhang, C., Liu, S., Zhao, P., Liu, H., \& Zhu, L. (2020). Pathological findings of COVID-19 associated with acute respiratory distress syndrome. The Lancet Respiratory Medicine. https://doi.org/10.1016/S2213-2600(20)30076-X

Yang, Z., Hao, J., Huang, S., Yang, W., Zhu, Z., Tian, L., Lu, Y., Xiang, H., \& Liu, S. (2020). Acute effects of air pollution on the incidence of hand, foot, and mouth disease in Wuhan, China. Atmospheric Environment, 225, 117358. https://doi.org/10.1016/j.atmosenv.2020.117358

Zaki, A. M., Van Boheemen, S., Bestebroer, T. M., Osterhaus, A. D., \& Fouchier, R. A. (2012). Isolation of a novel coronavirus from a man with pneumonia in Saudi Arabia. New England Journal of Medicine, 367(19), 1814-1820.

Zhang, X., Shi, M., Li, Y., Pang, R., \& Xiang, N. (2018). Correlating PM 2.5 concentrations with air pollutant emissions: A longitudinal study of the Beijing-Tianjin-Hebei region. Journal of Cleaner Production, 179, 103-113.

Zhao, J. L., \& Zhou, N. (2021). Impact of human health on economic growth under the constraint of environment pollution. Technological Forecasting and Social Change, 169, 120828. https://doi.org/10. 1016/j.techfore.2021.120828

Zhu, Y. J., Xie, J. G., Huang, F. M., \& Cao, L. Q. (2020). Association between short-term exposure to air pollution and COVID-19 infection: Evidence from China. Science of the Total Environment. https:// doi.org/10.1016/j.scitotenv.2020.138704

Zivot, E., \& Andrews, D. W. K. (1992). Further evidence of the great crash, the oil-price shock, and the unit root hypothesis. Journal of Business and Economic Statistics, 10, 251-270.

Publisher's Note Springer Nature remains neutral with regard to jurisdictional claims in published maps and institutional affiliations. 